\title{
Framing und Versicherheitlichung: Die diskursive Konstruktion des Klimawandels
}

In der Diskussion über die Versicherheitlichung der Umwelt, $d$. h. der Darstellung von Gefahren für die Umwelt als existenzielle Bedrohungen, nimmt der Klimawandel breiten Raum ein. Dabei wird oftmals postuliert, dass die globale Erwärmung direkt (in Form von Verteilungskonflikten) oder indirekt (z. B. über Migrationsströme) zu gewaltsamen Konflikten führt. Dieses Verständnis ist jedoch nur eine mögliche Lesart des Klimawandel-Sicherheits-Nexus. Wir problematisieren diese, indem wir die Theorie der Versicherheitlichung, wie sie in der Kopenhagener Schule entwickelt wurde, mit einem Framing-Ansatz verbinden. Die verschiedenen Frames von Klimawandel als Sicherheitsbedrohung unterscheiden sich dabei hinsichtlich der Frage, wen sie als Referenzobjekt annehmen und welche logischen Verknüpfungen sie zwischen Klimawandel und dessen Sicherheitsimplikationen vornehmen. Diese Problemdiagnosen bringen auch spezifische Politikempfehlungen hervor, was neues Licht auf das scheinbare Paradox wirft, dass zahlreiche Versuche, den Klimawandel zu versicherheitlichen, bisher nicht zu außergewöhnlichen Maßnahmen im Sinne der Kopenhagener Schule geführt haben.

Schlagworte: Versicherheitlichung, Klimawandel, Framing, Diskurs, Umweltsicherheit

\section{Klimawandel als Sicherheitsproblem}

"As climate change unfolds, one of its effects is a heightened risk of violent conflict « (Smith/Vivekananda 2007: 1). ${ }^{1}$ Dieses Zitat aus der Zusammenfassung eines Berichts für International Alert, einer von der Europäischen Union geförderten Nichtregierungsorganisation (NGO) zur Friedensarbeit, beschreibt treffend eine weit verbreitete Darstellung des Zusammenhangs von Umwelt und Sicherheit, die

1 Dieser Aufsatz wurde im Rahmen des Forschungsprojekts »Die Versicherheitlichung von Klimawandel: Akteure, Prozesse und Folgen (ClimaSec)« verfasst (http://www.wiso.uni-tuebingen.de/ faecher/ifp/lehrende/ipol/forschungsprojekte/climasec.html; letzter Zugriff: 25.9.2014). Das Projekt wird von der Deutschen Forschungsgemeinschaft (DFG) gefördert (Grant Nr. DI 1688/2-1). Eine frühere Version dieses Beitrags haben wir auf der gemeinsamen BISA/ISA-Konferenz in Edinburgh, 20. - 22.6.2012, vorgestellt. Unser Dank gilt den Kommentaren der TeilnehmerInnen an diesem Panel und der vier GutachterInnen der ZeFKo sowie Andreas Baur, Benno Keppner, Jerome Kuchejda, Hanna Spanhel, Sophie Veauthier und Josefa Velten für die Hilfestellung bei der Erarbeitung dieser Textfassung. 
sich in den letzten Jahrzehnten entwickelt hat und den Klimawandel mit einer erhöhten Konfliktwahrscheinlichkeit verknüpft. Es handelt sich hierbei um den Versuch, Klimawandel im Sinne der Kopenhagener Schule der Sicherheitsforschung $\mathrm{zu} »$ versicherheitlichen«, das heißt in einem Sprechakt eine existenzielle Bedrohung eines Referenzobjektes zu postulieren und damit außergewöhnliche Maßnahmen zur Beseitigung der Gefahr zu legitimieren (Buzan et al. 1998: Kap 4).

Wir greifen diese Arbeiten auf und argumentieren, dass die diskursive Verknüpfung von Klimawandel und gewaltsamen Konflikten nur eine mögliche Lesart der sicherheitspolitischen Implikationen des Klimawandels darstellt. Dieser Lesart werden wir zwei abweichende Konstruktionen des Klimawandel-Sicherheits-Nexus gegenüberstellen, nämlich 1.) die Versicherheitlichung von Klimawandel als existenzielle Bedrohung für die Menschheit als Ganze sowie 2.) die Repräsentation globaler Erwärmung als existenzielle Gefahr für das weltweite Ökosystem. Diese Konstruktionen unterscheiden sich nicht nur hinsichtlich der Frage, wer als Referenzobjekt angenommen wird, sondern auch darin, welche logischen Verknüpfungen sie zwischen globaler Erwärmung und deren Sicherheitsimplikationen vornehmen und welche Arten von außergewöhnlichen Maßnahmen gefordert werden. Sie werfen damit weitreichende politikrelevante und ethische Fragen auf.

Dabei entspricht die politische Bearbeitung des Klimawandels nicht den von Barry Buzan und seinen Kollegen (Buzan et al. 1998: Kap. 2) postulierten Notfallmaßnahmen (Methmann et al. 2013). Buzan und Ole Wæver (2009: 271-272) führen dies auf eine mangelnde Akzeptanz der Versicherheitlichungsversuche im Umweltsektor zurück. Andere AutorInnen hingegen verweisen auf diskursive Auseinandersetzungen über den genauen Klimawandel-Sicherheits-Nexus (Brzoska 2009; Detraz/Betsill 2009) und betonen, dass eine erfolgreiche (Trombetta 2008) bzw. konsistente (Methmann et al. 2013) Versicherheitlichung im Bereich des Klimawandels vielmehr zu einer politischen respektive technokratischen Problembearbeitung geführt hat, wenn auch nicht notwendigerweise auf globaler Ebene.

Wir unterscheiden uns von der bisherigen Literatur insofern, als wir die verschiedenen Varianten des Klimawandel-Sicherheits-Nexus systematischer herleiten und hierfür den Ansatz der Kopenhagener Schule mit dem Framing-Ansatz verbinden. Dies erlaubt uns, eine größere Bandbreite möglicher Versicherheitlichungen zu erkennen, und ermöglicht eine Analyse, die stärker die unterschiedlichen Akteure von Versicherheitlichung und die unterschiedlichen politischen Konsequenzen in Augenschein nimmt.

Um dieses Argument zu entwickeln, werden wir zunächst ausführen, wie ein Framing-Ansatz unserer Ansicht nach mit der Theorie der Versicherheitlichung verknüpft werden kann und diskutieren, welche Auswirkungen dies für das von der 
Kopenhagener Schule entwickelte Konzept der Versicherheitlichung hat. Darauf aufbauend werden wir drei konkurrierende Lesarten des Klimawandels als Sicherheitsbedrohung darstellen. Schließlich werden wir anhand einiger paradigmatischer Fälle illustrieren, wie eine Frame-geleitete Analyse neues Licht auf die Versuche, den Klimawandel zu versicherheitlichen, wirft und welche Ansatzpunkte es gibt, den (Miss-)Erfolg einzelner Framings in diesem Zusammenhang zu erklären.

\section{Das Framing von Versicherheitlichung}

Die Kopenhagener Schule definiert Versicherheitlichung als einen erfolgreichen Sprechakt, »through which an intersubjective understanding is constructed within a political community to treat something as an existential threat to a valued referent object, and to enable a call for urgent and exceptional measures to deal with the threat « (Buzan/Waever 2003: 491). Versicherheitlichung im Sinne der Kopenhagener Schule ist also ein Sprechakt, der bestimmte rhetorische Strukturen aufweist und spezifischen Regeln folgt. Dabei wird die Bedeutung von Sicherheit auf eine Freund-Feind Logik festgelegt, die außergewöhnliche Maßnahmen außerhalb des üblichen demokratischen Rahmens legitimiert. Dieses Verständnis von Sicherheit ist bereits in der Vergangenheit als ahistorisches Konzept kritisiert worden (Stritzel 2011), das die Rolle von Diskursen (Stritzel 2007) und gesellschaftlichen Kontexten (Balzacq 2005) negiert.

Entsprechend ihres Verständnisses von Sicherheit geht die Kopenhagener Schule (Buzan et al. 1998: 73) nicht davon aus, dass die Umwelt erfolgreich versicherheitlicht worden ist, da Versuche der Versicherheitlichung bisher lediglich »normale« politische Maßnahmen hervorgebracht hätten - und auch das nur in bescheidenem Maße, wie es etwa an den Auseinandersetzungen um das Kyoto-Protokoll und seine Fortsetzung deutlich wird -, sodass eher von einer Politisierung denn von einer erfolgreichen Versicherheitlichung von Umweltdegradation auszugehen sei. Das Label »Sicherheit« ist hingegen mit einer bestimmten Denkart und problematischen Praktiken verbunden, die keine Verhandlungen und politischen Debatten erlauben (Wæver 1995: 54). Von einer erfolgreichen Versicherheitlichung wird erst dann gesprochen, wenn das jeweils relevante Publikum von der Notwendigkeit solcher Maßnahmen überzeugt ist, wobei diese dann allerdings nicht tatsächlich ergriffen werden müssen (Buzan et al. 1998: Kap. 2). Eine erfolgreiche Versicherheitlichung setzt also voraus, dass Politiken außerhalb der normalen (demokratischen) Prozeduren stattfinden, wodurch das Risiko für eine Militarisierung und für feindliches Verhalten zunimmt (Eriksson 2001: 13). Die Kopenhagener Schule postuliert hinsichtlich dieser, aus einer realistischen Tradition entwickelten Sicherheitslogik eine 
oft problematisierte Beständigkeit des Verständnisses von Sicherheit (Williams 2003: 514-515). Eine solche Auffassung von Sicherheit als Extremsituation wurde als konzeptuell problematisch (Williams 2011; Trombetta 2008; Elbe 2005) und unzureichend für die Analyse empirischer Ausprägungen von Versicherheitlichung (Detraz/Betsill 2009; Trombetta 2008; 2011; Brzoska 2009) kritisiert.

Das scheinbare Paradox, dass die unzähligen und den Diskurs bestimmenden Versuche, den Klimawandel zu versicherheitlichen, bisher nicht zu außergewöhnlichen Maßnahmen im Sinne der Kopenhagener Schule geführt haben, wird unterschiedlich erklärt. Maria J. Trombetta (2011; 2008: 598) argumentiert, dass Versuche der Versicherheitlichung im Umweltsektor insoweit erfolgreich waren, als dass sie politische Maßnahmen zur Folge hatten, die andernfalls nicht realisiert worden wären. In ähnlicher Weise zeigen Rita Floyds Fallstudien (2008; 2010), dass nicht alle Versuche der Versicherheitlichung im Sinne der von der Kopenhagener Schule unterstellten konfrontativen Logik erfolgt sind. Vielmehr hat die Versicherheitlichung der Umwelt in Form verschiedener konkurrierender Lesarten von Umweltdegradation als Sicherheitsbedrohung stattgefunden (siehe auch Brzoska 2009; Detraz/Betsill 2009; McDonald 2013). Dies greift Maarten A. Hajers (1995) einflussreiche Arbeit über Umweltdiskurse auf, in welcher er feststellt, dass verschiedene Akteure Umweltprobleme sehr unterschiedlich definieren (siehe auch Litfin 1994) und dies immense politische Folgen hat. Anstatt konkurrierender Diskurse konstatieren Chris Methmann und Delf Rothe (2013) hingegen eine konsistente Lesart des Klimawandels als Apokalypse, die jedoch nicht zu Notfallmaßnahmen führt, sondern in eine technokratische Klima-Governance mündet. Allerdings scheint uns die Konzeptualisierung des Klimawandels als Apokalypse in dieser einheitlichen Form nicht zuzutreffen.

\subsection{Versicherheitlichung als Framing}

Während die Kopenhagener Schule ein formales Sprechakt-Modell von Versicherheitlichung vertritt, schlagen wir vor, Versicherheitlichung als Framing-Prozess zu verstehen. Im Gegensatz zu Angela Oels und Anabela Carvalho (2012), die aufbauend auf Fred Vultee (2010) Versicherheitlichung selbst als einen Frame begreifen, argumentieren wir, dass eine solche Konzeptualisierung von Versicherheitlichung als Framing Abweichungen von der durch die Kopenhagener Schule postulierten singulären sprachlichen Struktur erlaubt, sodass die Versicherheitlichung des Klimawandels eben keinen einheitlichen Frame darstellt. Ein Framing-Ansatz erfasst demnach die Diversität verschiedener Arten der Versicherheitlichung, beispielsweise im Umweltsektor, welche die Kopenhagener Schule nicht erklären 
kann, und vermeidet die theoretisch problematische Auffassung einer fixen Bedeutung von Sicherheit (Williams 2011; Stritzel 2011; 2007; Balzacq 2011; 2005; Huysmans 2006; Pram Gad/Petersen 2011).

Mit Erving Goffman definieren wir Frames als Interpretationsschemata, die Menschen dazu in die Lage versetzen, »to locate, perceive, identify and label [...] occurrences« (Goffman 1974: 21). Frames dienen also sowohl dazu, bestimmten Ereignissen Bedeutung zu verleihen, als auch, Interpretationen der Realität zu verdichten und zu vereinfachen. Dieser Prozess umfasst die Auswahl und Zuschreibung von Bedeutung. Frames heben somit bestimmte Interpretationen hervor und lenken die Aufmerksamkeit von anderen ab (Entman 1993).

Eine solche Framing-Perspektive erfasst die Versuche, ein Thema zu versicherheitlichen, nuancierter als das Instrumentarium der Kopenhagener Schule. In diesem Kontext ist eine Unterscheidung zwischen dem engeren Konzept der Frames und der umfassenderen Idee der Bilder (Images), wie sie in der Framing-Literatur vorgenommen wird, aufschlussreich. Ein einzelnes Bild kann, wie Kevin M. Carragee und Wim Roefs (2004: 218) in ihrer Analyse der Anti-Atomkraft-Bewegung zeigen, auf verschiedene Arten dargestellt werden, die in ihrer Ablehnung von Atomenergie übereinstimmen, zugleich aber unterschiedliche Begründungen für diese Position heranziehen. Wir argumentieren gleichermaßen, dass Sicherheit im Sinne der Kopenhagener Schule ein spezifisches Bild ist, das innerhalb eines bestimmten Rahmens verschiedene Lesarten der Bedrohung umfassen kann. In diesem Sinne ist die enge Konzeption von Sicherheit der Kopenhagener Schule zu restriktiv und hat dadurch die unterschiedlichen Darstellungen von Klimawandel als Sicherheitsbedrohung negiert. Unsere Argumentation, dass unterschiedliche Arten des Framing einer Sicherheitsbedrohung existieren, wird theoretisch (Balzacq 2011; Stritzel 2007; 2011; Guzzini 2011) und durch verschiedene Fallstudien untermauert. ${ }^{2}$

Die Framing-Perspektive auf Versicherheitlichung betont nicht nur die Möglichkeit unterschiedlicher diskursiver Darstellungen einer Sicherheitsbedrohung, sondern bietet sich auch als Ausgangspunkt für eine Analyse der Unterschiede dieser Lesarten an. Frames sind Deutungsrahmen, die komplexe Phänomene vereinfachen, Ereignissen so Sinn geben und Handlungen anleiten (Benford/Snow 2000: 614). Sie unterscheiden sich hinsichtlich der kausalen Mechanismen, die für die Beziehung zwischen Umwelt und Sicherheit postuliert werden, hinsichtlich der als gefährdet angenommenen Referenzobjekte und hinsichtlich der genauen Art der Bedrohung (zur ähnlichen Idee unterschiedlicher Grade von Versicherheitlichung vgl.

2 Vgl. hierzu Neal (2009) zu EU-Grenzpolitiken; Trombetta (2008; 2011) zu Umweltpolitiken und Salter (2008) zur kanadischen Behörde für Luftverkehrssicherheit. 
Adamides 2012: 104). Wessen Sicherheit durch den Klimawandel bedroht ist, hängt dabei eng mit der Problemdiagnose zusammen und hat auch Einfluss auf die daraus resultierenden Politikempfehlungen. Frames identifizieren also nicht nur ein als relevant erachtetes Problem, sondern benennen in diesem Zusammenhang auch Ursachen und Verantwortliche (Gerhards 1995: 228), was als diagnostische Dimension beschrieben wird. Darüber hinaus präsentieren sie in ihrer prognostischen Dimension oftmals eine plausible Lösung für das identifizierte Problem und bieten eine Begründung an, warum sich ein Engagement lohnt oder sogar notwendig ist (motivationales Framing, vgl. Gerhards/Rucht 1992: 582). Diese Dimensionen (siehe dazu auch Nisbet 2009: 15) sind so verschränkt, dass bestimmte Prognosen spezifische Politikempfehlungen implizieren (Benford/Snow 2000: 615; Hajer 1995: 6). Abhängig davon, wie beispielsweise der Klimawandel problematisiert wird, werden verschiedene Handlungsmöglichkeiten betont (Hulme 2010: 172).

Während Versuche der Versicherheitlichung, wie sie von der Kopenhagener Schule beschrieben werden, auch eine diagnostische Dimension (existenzielle Bedrohung eines Referenzobjekts) und eine damit verbundene Handlungsanweisung (Notfallmaßnahmen) umfassen, hebt unser Framing-Ansatz hervor, dass sich Ansätze der Versicherheitlichung darin unterscheiden, wie diese Befunde zu interpretieren sind - also hinsichtlich der (kausalen) Verbindung, die zwischen der Bedrohung, dem bedrohten Referenzobjekt und den vorgeschlagenen Maßnahmen hergestellt wird (Rein/Schön 1991: 262; Hajer 1995: 56). Dementsprechend setzt die diagnostische Dimension eine Schuldzuschreibung voraus (Benford/Snow 2000: 616). Veränderungen der präzisen Identifikation und der Beschreibung eines Problems beeinflussen die Art und Weise, wie die Adressaten der Versicherheitlichung die Situation interpretieren (Scheufele 2000). Die so getroffene Zuschreibung von Verantwortung für die entsprechende Sicherheitsbedrohung legt letztlich auch die zu treffenden Maßnahmen nahe.

Diese Konzeptualisierung von Versicherheitlichung ermöglicht es, den Gedanken einer existenziellen Bedrohung beizubehalten und dennoch zu erklären, wie Abweichungen von der fixen Struktur von Sicherheit als Extremsituation dazu führen, dass unterschiedliche außergewöhnliche Maßnahmen eingefordert werden, die nicht alle auf einen nicht mehr demokratisch kontrollierten Ausnahmezustand abzielen. Wenn also beispielsweise die existenzielle Bedrohung darin liegt, dass die genauen Folgen des Klimawandels für die menschliche Sicherheit unabsehbar sind, dann besteht die außergewöhnliche Natur der geforderten Maßnahmen darin, dass ein Handeln der internationalen Gemeinschaft selbst in der Abwesenheit von exaktem Wissen über die Folgen globaler Erwärmung gefordert und akzeptiert wird. Im Gegensatz zu Methmann und Rothe (2013: 114-116) betrachten wir dies als 
Forderung nach außergewöhnlichen Maßnahmen, der jedoch eine andere Problemdiagnose zugrunde liegt als dem Ruf nach Geoengineering (Copper 2006) oder Grünhelmen (Malone 1996). Exemplarisch hierfür steht auch die Diskussion über die Anerkennung des »Klimaflüchtlings « als neue rechtliche Kategorie (Biermann/ Boas 2008). Während dies im klassischen Sinne keine Notfallmaßnahme außerhalb des demokratischen Rahmens ist, stellt dieser Schritt dennoch eine qualitative neue Stufe der rechtlichen Problembearbeitung dar, die ohne den Versuch, Klimamigration zu versicherheitlichen, so nicht stattgefunden hätte (Bettini 2013: 128).

Ein Fokus auf die Betrachtung der Problemdiagnose, einschließlich des (kausalen) Zusammenhangs, der zwischen dem Problem und seinen Auswirkungen hergestellt wird, erweitert und modifiziert die Auffassung der Kopenhagener Schule, dass auf die Präsentation von etwas als existenzieller Bedrohung notwendigerweise ein Ruf nach eng definierten außergewöhnlichen Maßnahmen folgt (Buzan et al. 1998: 20). Die Sicherheitsimplikationen des Klimawandels werden für so unterschiedliche Opfer wie besonders verletzliche menschliche Gruppen, (Insel-)Staaten, deren gesamte Existenz bei einem steigenden Meeresspiegel gefährdet ist, und den gesamten Planeten diskutiert (Methmann/Rothe 2012: 110). Inwiefern die fortschreitende globale Erwärmung für diese Referenzobjekte eine existenzielle Gefährdung darstellt, unterscheidet sich jedoch hinsichtlich der zugrunde liegenden Bedrohungslogik fundamental. Demnach ist zentral, wie und nicht $o b$ ein Problem als existenzielle Bedrohung für ein bestimmtes Referenzobjekt präsentiert wird und warum es zunächst als Problem wahrgenommen wurde. Die Verbindung zwischen der Problemdiagnose (existenzielle Bedrohung) und der politischen Reaktion (außergewöhnliche Maßnahmen), die im Rahmen der Kopenhagener Schule unanfechtbar erscheint, kann demnach buchstäblich entflochten werden (vgl. Balzacq 2011). Das scheinbare Paradox eines apokalyptischen Diskurses (Methmann/Rothe 2012) über die Sicherheitsimplikationen des Klimawandels ohne darauf aufbauende außergewöhnliche Maßnahmen wird dadurch aufgelöst.

\subsection{Frame-Transformationen und Frame-Auseinandersetzungen}

Darüber hinaus betont ein Framing-Ansatz, wie sich unterschiedliche Arten der Konstruktion einer Sicherheitsbedrohung entwickeln, wie sie miteinander konkurrieren und wie sie sich im Laufe der Zeit verändern. Die Theorie der Versicherheitlichung mit ihrem fixen Verständnis von Sicherheit ist nicht in der Lage, die Entstehung verschiedener Sicherheitslogiken - beispielsweise durch die Versicherheitlichung nicht-traditioneller Themen wie dem Klimawandel - zu erfassen (Trombetta 2011). Framing hingegen ist ein inhärent dynamischer Prozess (Benford/Snow 2000: 614), der auch die Konstruktion neuer Frames umfasst, die sich 
nicht nur von den existierenden unterscheiden, sondern diese auch infrage stellen. Robert D. Benford und David A. Snow (2000) beschreiben, wie sich Frames im Rahmen von drei teilweise überlappenden Prozessen entwickeln, die sie als diskursiv, strategisch und konfliktiv charakterisieren.

Diskursive Auseinandersetzungen hinsichtlich der verschiedenen Lesarten des Klimawandel-Sicherheits-Nexus sind bereits verschiedentlich beleuchtet worden (Trombetta 2008; Brzoska 2009; Detraz/Betsill 2009), wobei die AutorInnen überwiegend offen lassen, welche Akteure bestimmte Probleme (strategisch) diagnostizieren (vgl. Schmidt 2012: 73). Zwei Subkategorien dieser Prozesse sind von besonderem Interesse, um aufzuzeigen, wie Versicherheitlichung als dynamischer Prozess neu definiert werden kann. Das Phänomen der Frame-Transformation bezieht sich auf die Veränderung alter Auffassungen oder das Erzeugen neuer Bedeutungen, um Argumentationslinien für eine bestimmte Zielgruppe vertrauter und somit besser zugänglich zu präsentieren. Demzufolge können Versuche, ein Thema $\mathrm{zu}$ versicherheitlichen, unterschiedliche Formen annehmen, um bestimmten institutionellen Kontexten zu entsprechen (Salter 2011; Léonard/Kaunert 2011) oder neu verhandelt werden, weil Akteure erkennen, dass sie der grundlegenden Terminologie - beispielsweise derjenigen der Sicherheit - nicht entkommen, aber ihre Bedeutung (teilweise) neu definieren können (Reese 2007: 153).

Darüber hinaus zeigt die Untersuchung von Frame-Auseinandersetzungen (Benford 1993), wie unterschiedliche Lesarten einer Bedrohung einander beeinflussen. Frame-Auseinandersetzungen bezeichnen Situationen, in denen Konflikte über die Lesart eines Phänomens innerhalb eines spezifischen Paradigmas entstehen. Diese Erkenntnis kann auf die Untersuchung von Versicherheitlichungsprozessen angewandt werden, bei denen verschiedene Lesarten einer bestimmten Bedrohung beispielsweise Umweltzerstörung - interagieren und konkurrieren. Die Feststellung, der Klimawandel sei eine Sicherheitsbedrohung - das Klimawandel-Sicherheits-Bild - kann beispielsweise so unterschiedlich beschrieben werden, dass eine Auseinandersetzung über die Frage entsteht, welche Darstellung der Sicherheitsimplikationen des Klimawandels das Phänomen am besten erfasst.

Eine Konzeptualisierung von Versicherheitlichung als Framing wirft die Frage auf, ob die Abweichung vom Verständnis von Sicherheit als genau definiertem Sprechakt nicht in eine zu amorphe und zu breite Definition von Sicherheit mündet, die konträr zu den Zielen der Kopenhagener Schule steht (Buzan et al. 1998: 1-4), und ob daraus resultierende Konzeptualisierungen von Sicherheit daher noch unter die Theorie der Versicherheitlichung subsumiert werden können (Stritzel 2007; Williams 2011; Huysmans 1998; Salter 2011; Sjostedt 2011). Der von der Kopenhagener Schule vertretene Formalismus stellt jedoch nur eine der möglichen Antworten auf die methodologische Herausforderung dar, Ansätze der Versicherheitlichung zu untersuchen, die unterschiedlichen Sicherheitslogiken folgen, ohne analytische Genauigkeit und Trennschärfe einzubüßen (Williams 2011). Das von uns 
vertretene Verständnis von Versicherheitlichung als Framing ermöglicht die Identifikation unterschiedlicher Arten der Versicherheitlichung von Klimawandel, wobei die jeweiligen Frames an der grundlegenden Idee der Versicherheitlichung als Konstruktion einer existenziellen Bedrohung mit Politikfolgen festhalten, die Bedrohung aber unterschiedlich konstruieren, sich auf unterschiedliche Referenzobjekte beziehen und unterschiedliche Maßnahmen propagieren. So bewahrt der Framing-Ansatz die analytische Trennschärfe von Sicherheit als Konstruktion einer existenziellen Bedrohung, bietet aber gleichzeitig ein analytisches Werkzeug, um zu untersuchen, wie genau diese Bedrohungen konstruiert werden und was sie unterscheidet. Die Analyse unterschiedlicher Frames macht deutlich, wann Sicherheitspraktiken auftreten, die der von der Kopenhagener Schule vorgestellten Logik folgen, erfasst aber auch Sicherheitslogiken, die nicht vollständig dem von der Kopenhagener Schule verfochtenen Sicherheitsverständnis entsprechen.

\section{Die drei Frames der Versicherheitlichung des Klimawandels}

Das veränderte Verständnis von Versicherheitlichung als Framing ermöglicht es, verschiedene Arten der Versicherheitlichung des Klimawandels zu identifizieren, in denen Frames an der grundlegenden Form der Versicherheitlichung als Darstellung einer existenziellen Bedrohung festhalten, aber die Bedrohung unterschiedlich konstruieren. Die Kategorisierung der Frames, die einen Klimawandel-SicherheitsNexus postulieren, erfolgt anhand zweier, in der Literatur als wichtige Unterscheidungsmerkmale identifizierter Elemente. Dies ist einerseits das Referenzobjekt: Page (2010: 3) unterscheidet zwischen einer oberflächlichen entmilitarisierten Vorstellung von Sicherheit (Klimawandel als Bedrohung für staatliche Sicherheit) und einer stark entmilitarisierten Vorstellung, die auf das Konzept der menschlichen Sicherheit Bezug nimmt und somit die Folgen für das Individuum in den Mittelpunkt stellt. Rothe (2010) differenziert in ähnlicher Weise zwei Lesarten des Klimawandel-Konflikt-Nexus: eine, die Klimawandel als Feind der Menschheit als Ganze porträtiert, und eine zweite, die dessen Folgen für die Sicherheit von (Entwicklungs-)Ländern in den Mittelpunkt rückt. Wir folgen hierbei dem Vorschlag von Matt McDonald (2013), der sich in seiner Kategorisierung ebenfalls vorrangig auf Unterschiede im Referenzobjekt bezieht.

Andererseits unterscheiden sich die Frames in der Problemdiagnose. Nicole Detraz und Michele Betsill (2009) richten ihr Augenmerk auf zwei vergleichbare Arten, wie Klimawandel und Sicherheit in der Debatte des Sicherheitsrats der Vereinten Nationen 2007 in Zusammenhang gebracht wurden: als Umweltkonflikt und Umweltsicherheit. Auch Trombetta (2011) bezieht sich auf zwei Entwicklungen in der Debatte um Umweltsicherheit: einen Diskurs zu den nationalen Sicherheitsimplikationen und eine Lesart, die Umweltzerstörung als eine Bedrohung für die globale Ordnung und 
Stabilität charakterisiert. Dies hängt eng zusammen mit den drei in der Klimawandeldebatte diskutierten Konzepten Bedrohung, Risiko und Resilienz.

Ähnlich wie die genannten AutorInnen die Identifikation der verschiedenen Lesarten der Sicherheitsdimension der globalen Erwärmung nicht nur an theoretische Erwägungen knüpfen, spielen auch bei unserer Herleitung und Unterscheidung der drei relevanten Frames Policy-Dokumente und Diskussionspapiere von Think Tanks eine wichtige Rolle. Die Frames wurden also, angelehnt an retroduktive Verfahren (Glynos/Howarth 2007: Kap. 1), durch einen permanenten Dialog zwischen Theorie und empirischem Material entwickelt. ${ }^{3}$ Dabei erheben wir nicht den Anspruch auf eine vollständige Abdeckung der Debatte; vielmehr verstehen wir unseren Aufsatz als Beitrag zur Ermöglichung umfassenderer Analysen. Insofern konzentriert sich unser Textkorpus auf Dokumente, die um das Jahr 2007 erschienen sind, als die Aufmerksamkeit für die Folgen des Klimawandels einen Höhepunkt erreichte (Neverla/Schäfer 2010). Wir stützen uns in erster Linie auf sechs USamerikanische Dokumente, die das gesamte Spektrum der in der Literatur diskutierten möglichen diskursiven Verknüpfungen von Klimawandel und Sicherheit abdecken sowie zusätzlich auf ein Dokument aus dem deutschen Kontext, das als einflussreichstes Beispiel der Thematisierung von Klimawandel als Problem für die menschliche Sicherheit gilt (Brzoska 2009). ${ }^{4}$

Der Korpus ist thematisch organisiert (vgl. Busse/Teubert 1994: 14) und die vergleichsweise geringe Anzahl der Dokumente trägt der Beobachtung von Siegfried Jäger (2006: 103) Rechnung, dass die Bandbreite diskursiver Artikulationen zu einem bestimmten Zeitpunkt in der Regel erstaunlich begrenzt ist. Nichtsdestotrotz ermöglicht unsere exemplarische Analyse vor allem eine kritische Lektüre prominent diskutierter Dokumente, die zeigt, dass sich diese Lesarten des KlimawandelKonflikt-Nexus anhand der analytischen Kategorien Problemperzeption, Referenzobjekt und Lösungsvorschläge strukturieren lassen (Buck 2013), statt umfassend die diskursive Verknüpfung von Klimawandel und Sicherheit in verschiedenen

3 Ein retroduktives Vorgehen basiert auf dem ständigen Austausch zwischen Theorie und Empirie, wobei aus dem kontinuierlichen Zusammenspiel zwischen theoriegeleiteter und empirischer Kategorienbildung der Prozess paralleler Theoriegenerierung und -überprüfung resultiert. Retroduktion bezeichnet demzufolge einen interpretativen Analyseprozess, in dessen Rahmen die »traditionellen« Grenzen zwischen induktivem und deduktivem Vorgehen aufgelöst werden.

4 Bei den ausgewählten Dokumenten handelt es sich um Busby et al. (2010); Busby (2007); Campbell et al. (2007); CNA (2007); Levy et al. (2008); Schwartz/Randall (2003) und WBGU (2007). Diese Dokumente wurden ausgewählt, da sie in der wissenschaftlichen Auseinandersetzung mit der diskursiven Konstruktion des Klimawandels als Sicherheitsbedrohung als typische Fälle für bestimmte Argumentationsmuster identifiziert wurden und außerdem die öffentliche Debatte auf besondere Weise geprägt haben. Ihr Erscheinungszeitraum ermöglicht darüber hinaus, den Diskurs rund um das Jahr 2007 nachzuzeichnen, das aufgrund der Debatte im Sicherheitsrat der Vereinten Nationen $\mathrm{zu}$ den Sicherheitsimplikationen des Klimawandels und der Veröffentlichung des fünften Berichts des Weltklimarates wegweisend war. 
nationalen Kontexten nachzuvollziehen. Zunächst haben wir nach in der Sekundärliteratur im Zusammenhang mit bestimmten Argumentationsmustern genannten Schlüsselbegriffen gesucht und deren Verwendung erfasst, was aufgrund der wenigen Dokumente ohne computergestützte Software erfolgte. ${ }^{5}$ Anschließend wurde der Kontext dieser Artikulationen in den sieben Dokumenten genauer betrachtet, was zur Aufnahme weiterer Schlüsselbegriffe in unten stehende Tabelle 1 auf Grundlage der Dokumentenanalyse führte. Auf dieser Basis unterscheiden wir zwischen einem Umweltkonflikt- und einem Umweltsicherheits-Frame, denen wir den, in der bisherigen Debatte marginalisierten Frame der Ökologischen Sicherheit gegenüberstellen. Diese sollen im Folgenden näher ausgeführt werden.

\subsection{Umweltkonflikte}

Der Umweltkonflikt-Frame lässt sich zu den Studien von Richard Ullmann (1983) und Thomas Homer-Dixon (1994; 1999) zurückverfolgen und wurde in den 2000er Jahren in den USA zunehmend von Akteuren artikuliert, die Aufmerksamkeit für die drohende Klimakatastrophe generieren wollten, indem sie das Narrativ nationaler Sicherheit bedienten und auf diese Weise Klimapolitik zu neuer Aufmerksamkeit unter der Bush-Administration verhalfen (Richert 2011).

Kern dieser Argumentation ist die These, dass Ressourcenknappheit einen signifikanten Einfluss auf den Ausbruch und die Intensität bewaffneter Konflikte zwischen und innerhalb von Staaten hat, während aufgrund der neo-malthusianischen Prägung dieser Argumentation die Möglichkeit einer engeren Kooperation angesichts von Ressourcenknappheit negiert wird (Verhoeven 2011: 695). Zusätzlich zu Studien, die versuchen, eine direkte Verbindung zwischen Umweltveränderungen und Gewaltkonflikten aufzuzeigen (Burke et al. 2009; Zhang et al. 2007), sowie den damit verbundenen Diskussionen über Ressourcenkriege (Ross 2004) haben einige WissenschaftlerInnen intervenierende Faktoren identifiziert, die die Wahrscheinlichkeit für einen umweltbedingten Konflikt erhöhen, wie zum Beispiel Migration und Bevölkerungswachstum (Barnett 2001). Insbesondere Debatten über »Wellen« von Flüchtlingen, die aufgrund klimainduzierter Knappheit den globalen Norden »überrennen« werden, greifen malthusianische Deutungsmuster bzw. Degradationsnarrative auf (Hartmann 2009; 2013).

5 Aufbauend auf das beschriebene Vorgehen zur Unterscheidung der Frames wurde nach Schlüsselbegriffen, die auf bestimmte Referenzobjekte verweisen (Individuum, Menschheit, (National-)Staat, Ökosystem) sowie Begriffen, die den Zusammenhang zwischen Klimawandel und Sicherheitsbedrohung spezifizieren (Krieg, (bewaffneter) Konflikt, Sicherheit, Vulnerabilität) gesucht. 
Die Thematik der Umweltkonflikte wird durch ein enges Verständnis der Beziehung zwischen Sicherheit und Umwelt dominiert. Es wird hauptsächlich die Gefahr heraufbeschworen, dass sich bestimmte Gemeinschaften, allen voran Staaten, aufgrund von Umweltzerstörung an gewaltsamen Konflikten beteiligen (Detraz/Betsill 2009: 305). Nichtmilitärische Bedrohungen werden in der Debatte nur in dem Maße berücksichtigt, in dem sie die Sicherheit von Staaten gefährden. Exemplarisch für diese Lesart des Klima-Konflikt-Nexus steht Homer-Dixons Analyse interner Ressourcenkonflikte, die ihm zufolge im Endeffekt den Staat (und nicht einzelne Leben oder die menschliche Sicherheit) gefährden (vgl. auch Kaplan 1994 für eine ähnliche Argumentation).

Mit einem solchen Verständnis der Verknüpfung von Klimawandel und Konflikt gehen spezifische Politikempfehlungen einher, die gewöhnlich auf eine Stärkung traditioneller nationaler Sicherheitsinstrumente abzielen (Brzoska 2009: 144). Folglich richten sich solche Vorschläge in erster Linie an den Staat. Darüber hinaus heben Politikempfehlungen innerhalb des Paradigmas von Umweltkonflikten die Bedeutung von Anpassungen an den Klimawandel hervor, sofern Staaten das Risiko umweltbedingter Konflikte reduzieren wollen. Ein solcher Fokus auf Anpassung anstatt Vermeidung von Klimawandel engt nicht nur die Auswahl an möglichen Politikempfehlungen ein (Detraz/Betsill 2009: 306), sondern baut auf einem engen Verständnis von Anpassung auf, welches auf die Teile der Bevölkerung abzielt, die am ehesten in (Ressourcen-)Konflikte involviert sind (vgl. Ross 2003). Gleichzeitig schützt ein solcher Fokus auf nationale Sicherheitspolitik die Hauptopfer der globalen Erwärmung nicht (Nordås/Gleditsch 2007: 635).

\subsection{Umweltsicherheit}

Die Idee der Umweltsicherheit wurde in den 1970er und 1980er Jahren in den Brandt- und Brundtland-Berichten entwickelt und wurde zunächst vor allem von Akteuren propagiert, die eine Erweiterung des Sicherheitsbegriffs befürworteten (für eine Zusammenfassung siehe Daase 2009). Zu Beginn des neuen Jahrtausends gewann der entsprechende Frame auch als Gegendiskurs zur befürchteten Verengung der Debatte auf »Klimakriege« an Bedeutung.

Der entsprechende Frame hebt die nachteiligen Effekte von Umweltzerstörung für die Menschheit als Ganze und das Gemeinwohl hervor, anstatt die Analyse auf Kriege zwischen oder innerhalb von Staaten zu begrenzen (Detraz/Betsil 2009: 306; Smith 2010: 233). Durch den Fokus auf menschliche Verletzlichkeit (vgl. Vogel/ O'Brien 2004; Adger 2006; Eakin/Luers 2006) und den Einfluss von Umweltveränderungen auf das Individuum grenzt sich dieser Diskurs von staatszentrierten 
Umweltkonfliktansätzen ab und ist eng verknüpft mit dem Konzept der menschlichen Sicherheit. Der Umweltsicherheits-Frame stellt also die Folgen von Klimawandel für die gesamte Menschheit bzw. die Individuen in den Mittelpunkt der Problemdiagnose und daraus resultierender Politikempfehlungen.

Im Gegensatz zum Fokus auf Anpassung innerhalb des Umweltkonfliktdiskurses betonen Empfehlungen mit Bezug zur Umweltsicherheit die Relevanz präventiver Maßnahmen (Trombetta 2008: 594) und langfristiger Ziele, um Umweltveränderungen zu begegnen (Detraz/Betsill 2009: 308) und priorisieren menschliche gegenüber staatlicher Sicherheit. Hinsichtlich des Klimawandels gelten die Reduktion von Treibhausgasen sowie Adaptation als zentral. Deren Ausbleiben wird entsprechend als existenzielle Bedrohung für die menschliche Sicherheit gewertet (Brzoska 2009: 142). Dabei argumentiert Corry (2012), dass der Begriff des »Risikos«, der dem Plädoyer für präventive Maßnahmen zugrunde liegt, und das Konzept der Bedrohung zwei qualitativ unterschiedliche Logiken darstellen, wobei Erstere nicht zu Versicherheitlichung führt. Demgegenüber zeigt eine Framing-Perspektive, dass der Umweltsicherheits-Frame verschiedene Aspekte einer durch den Risikobegriff dominierten Problembearbeitung des Klimawandels wie vorbeugende Maßnahmen beinhaltet, ohne von der Identifikation einer existenziellen Gefährdung - in diesem Fall für die menschliche Sicherheit - abzurücken.

Der Umweltsicherheits-Frame überschneidet sich unter Umständen mit dem Umweltkonflikt-Frame, wenn beispielsweise konstatiert wird, dass sich Umweltkonflikte als umweltbedingte, gewaltsame Konflikte nicht bloß auf die Stabilität eines Staates, sondern auch auf das Wohlergehen von Individuen auswirken. Dennoch unterscheiden sich die beiden Frames fundamental voneinander, da Staaten im Frame Umweltkonflikt das zentrale, zu schützende Referenzobjekt sind, während sie im Frame Umweltsicherheit als Akteure dargestellt werden, welche sich auf eine Art und Weise verhalten, die die menschliche Umwelt-Sicherheit gefährdet (Dalby 2000: 2).

\section{3 Ökologische Sicherheit}

Zusätzlich zu diesen beiden Frames haben wir - wie auch McDonald (2013) - einen dritten potenziellen Frame der ökologischen Sicherheit identifiziert. Dessen Betonung von Interdependenz und Symbiose verweist auf die Ursprünge des Konzeptes, das auf den in den 1970er Jahren entwickelten Ideen von komplexer und systemischer Ökologie beruht. Crawford S. Holling (1973) entwickelte die Idee der KoEvolution von Mensch und Ökologie als ein komplexes, integriertes System. In aktuellen Debatten über die Sicherheitsimplikationen des Klimawandels wurde 
dieser Frame jedoch bisher marginalisiert, wie auch eine Betrachtung des Korpus zeigt. Lediglich in einer Studie wird der Klimawandel auch als Gefahr für Ökosysteme thematisiert und die Interdependenz menschlichen Lebens und natürlicher Umgebung betont (Busby et al. 2010: 26-28).

Das Konzept der ökologischen Sicherheit sieht die Menschheit als Teil eines gröBeren Ganzen und konzentriert sich dementsprechend auf die holistisch gedachte Umwelt als Hauptreferenzobjekt. Während der »Diskurs ökologischer Sicherheit« (Walker/Cooper 2011) die Interdependenz von Mensch und Umwelt in den Vordergrund stellt, betonen neuere Strömungen im Sinne von Rosalie Bertells (2001) Konzept der Umweltsicherheit die Rolle der natürlichen Umwelt als solche anstatt als Lebensgrundlage für den Menschen und stellen ein intaktes Ökosystem somit über andere menschliche oder staatliche Referenzobjekte.

Der Frame folgt der Idee einer komplexen Ökologie, die von Erika Cudworth und Stephen Hobden (2010) sowie von Simon Dalby (1992) entwickelt wurde. Sie betonen die gegenseitige Abhängigkeit und Symbiose verschiedener Elemente innerhalb des globalen Ökosystems und stellen dementsprechend den Glauben an eine technologisch-institutionelle Bewältigung der gegenwärtigen Probleme infrage (Cudworth/Hobden 2010: 8). Dies stellt einen zentralen Unterschied zum Umweltsicherheits-Frame dar, in dessen Rahmen die Chancen neuer Governance-Formen sowie technologischer Neuerungen zur Verhinderung des Klimawandels und der Anpassung prominent diskutiert werden. Diese Lesart von Klimawandel als existenzielle Bedrohung für die ökologische Sicherheit zielt auf die Restrukturierung und Transformation risikoverursachender Aktivitäten ab, statt den Schutz einzelner Staaten, Gruppen oder Menschen in den Mittelpunkt zu stellen. Der damit eng verwandte Diskurs ökologischer Resilienz lehnt auf ähnliche Weise den Schutz einzelner Gruppen oder materieller Errungenschaften ab und fordert stattdessen Engagement im Sinne der oben genannten Prinzipien, wobei Handlungslogiken des kapitalistischen Systems infrage gestellt werden (MacKinnon/Derickson 2013).

\section{Politische Prozesse der Versicherheitlichung}

Ein Framing-Ansatz erschöpft sich nicht in der Analyse verschiedener Frames, sondern rückt vielmehr den politischen/diskursiven Prozess der Behandlung von Klimawandel in Bezug auf seine Sicherheitsimplikationen in den Mittelpunkt. Welche Frames politische Bedeutung erlangen, hängt dabei nicht nur von der Kohärenz und Plausibilität der Problemdiagnose ab, sondern auch von den Akteuren, die diese vertreten, und ihrer Interaktion mit dem Publikum. Anhand paradigmatischer Fälle (Flyvbjerg 2006) soll im Folgenden diskutiert werden, welche Akteure bestimmte 
Frames verbreiten, unter welchen Umständen diese Eingang in die politische Debatte finden können und welche Folgen dies möglicherweise nach sich zieht.

Tabelle 1: Frames zu Klimawandel und Sicherheit

\begin{tabular}{|c|c|c|c|c|}
\hline $\begin{array}{l}\text { Frame/ } \\
\text { Diskurs }\end{array}$ & $\begin{array}{l}\text { Referenz- } \\
\text { objekt }\end{array}$ & $\begin{array}{l}\text { Diagnose- } \\
\text { Dimension }\end{array}$ & $\begin{array}{l}\text { Prognose- } \\
\text { Dimension }\end{array}$ & $\begin{array}{l}\text { Zugehörige } \\
\text { Schlüsselbegriffe }\end{array}$ \\
\hline $\begin{array}{l}\text { Umwelt- } \\
\text { konflikt }\end{array}$ & $\begin{array}{l}\text { Bestimmte } \\
\text { Gemeinschaf- } \\
\text { ten, vor allem } \\
\text { Staaten }\end{array}$ & $\begin{array}{l}\text { Fokus auf gewaltsame } \\
\text { Konflikte, wenn natür- } \\
\text { liche Ressourcen knapp } \\
\text { werden; zentrale Rolle } \\
\text { des Militärs; Umwelt } \\
\text { als endliche Ressource }\end{array}$ & $\begin{array}{l}\text { Kurzfristige Maßnah- } \\
\text { men; eher Anpassung } \\
\text { und reaktive (militäri- } \\
\text { sche) Maßnahmen }\end{array}$ & $\begin{array}{l}\text { Ressourcensicher- } \\
\text { heit, Ressourcen- } \\
\text { konflikte, Degrada- } \\
\text { tion natürlicher Res- } \\
\text { sourcen, Wasser- } \\
\text { konflikte, Energie- } \\
\text { sicherheit und Di- } \\
\text { versifizierung, mili- } \\
\text { tärische Maßnahmen }\end{array}$ \\
\hline $\begin{array}{l}\text { Umwelt- } \\
\text { sicherheit }\end{array}$ & $\begin{array}{l}\text { Gesamte } \\
\text { Menschheit, } \\
\text { Individuum }\end{array}$ & $\begin{array}{l}\text { Alltägliche Sicherheits- } \\
\text { implikationen für alle } \\
\text { Menschen; Fokus auf } \\
\text { menschliche Vulnera- } \\
\text { bilität aufgrund von } \\
\text { Umweltveränderungen; } \\
\text { Umwelt als gemeinsa- } \\
\text { mes Gut }\end{array}$ & $\begin{array}{l}\text { Langzeitstrategien zur } \\
\text { Bekämpfung von Um- } \\
\text { weltveränderungen; } \\
\text { Vermeidung und prä- } \\
\text { ventive Maßnahmen } \\
\text { zusätzlich zu Adapta- } \\
\text { tionsstrategien }\end{array}$ & $\begin{array}{l}\text { Menschliche Sicher- } \\
\text { heit, globale Sicher- } \\
\text { heit, Klima als ge- } \\
\text { meinsames Gut, } \\
\text { menschliche Ver- } \\
\text { letzlichkeit, Infra- } \\
\text { struktur globalen } \\
\text { Regierens }\end{array}$ \\
\hline $\begin{array}{l}\text { Ökologische } \\
\text { Sicherheit }\end{array}$ & $\begin{array}{l}\text { Umwelt oder } \\
\text { Ökosystem als } \\
\text { Ganzes }\end{array}$ & $\begin{array}{l}\text { Menschliche Einbet- } \\
\text { tung in das globale } \\
\text { Ökosystem; Gefahr für } \\
\text { die Umwelt als solche } \\
\text { (einschl. Pflanzen und } \\
\text { Tiere), Umwelt als zu } \\
\text { schützendes Gut }\end{array}$ & $\begin{array}{l}\text { Wechsel von Sicher- } \\
\text { heit zu Risikominde- } \\
\text { rung; Restrukturie- } \\
\text { rung risikoverursa- } \\
\text { chender Aktivitäten } \\
\text { statt dem Versuch, } \\
\text { bestimme Gruppen } \\
\text { durch Vermeidung } \\
\text { von oder Anpassung } \\
\text { an Umweltverände- } \\
\text { rungen zu schützen }\end{array}$ & $\begin{array}{l}\text { Ökosystem, Gren- } \\
\text { zen von Wachstum, } \\
\text { Mensch-Natur- } \\
\text { Beziehungen, ge- } \\
\text { genseitige Abhän- } \\
\text { gigkeit, Symbiose, } \\
\text { Risiko }\end{array}$ \\
\hline
\end{tabular}

Quelle: Eigene Darstellung

\subsection{Akteure}

Im Gegensatz zum üblichen Schwerpunkt auf Versicherheitlichung durch politische Eliten (Buzan et al. 1998) zeigt die Debatte über die Sicherheitsimplikationen des Klimawandels, dass Versuche der Versicherheitlichung im Umweltsektor durch eine Vielzahl nicht- oder teilstaatlicher Akteure und Institutionen erfolgen (Neverla/ Schäfer 2012). Versicherheitlichungen der Umwelt werden oftmals von NGOs und Think Tanks artikuliert (Brzoska 2009), bevor sie von politischen Eliten aufgegriffen 
werden (für eine ähnliche Beobachtung im Fall des Ozonregimes vgl. Trombetta 2011). Die globale Erwärmung und ihre Folgen sind ein zentrales Thema der Öffentlichkeitsarbeit einiger NGOs (Neverla/Schäfer 2012: 20), wobei diese in ihrer Argumentation nicht nur bestehende kulturelle Repertoires und Metadiskurse aufgreifen, sondern auch als "producers of new meanings « (Tarrow 1982: 189) fungieren. Daneben spielen auch mehr oder weniger unabhängige Think Tanks eine große Rolle in der Diskussion über die Sicherheitsimplikationen des Klimawandels. Unterschiedliche Akteure verfolgen dabei unterschiedliche Strategien: Während Think Tanks auf access-Strategien, also den Zugang zu relevanten politischen Entscheidungsträgern setzen, bemühen sich NGOs oftmals, ihre Problemanalysen und Handlungsempfehlungen im Sinne einer voice-Strategie durch spektakuläre Aktionen in die Öffentlichkeit zu tragen (Schmidt 2012: 82-84).

Eine Analyse der oben genannten Dokumente zeigt einen Zusammenhang zwischen den Akteuren, die bestimmte Dokumente verfasst haben oder in den Beratungsprozess involviert waren, sowie dem dominanten Framing des KlimawandelSicherheits-Nexus. Die Dominanz des Umweltsicherheits-Frames in Studien, die unter Beteiligung (ehemaliger) Militärs entstanden sind (Campbell et al. 2007; CNA 2007) oder vom US-Verteidigungsministerium in Auftrag gegeben wurden (Schwartz/Randall 2003), im Gegensatz zu Verweisen auf die Idee der Umweltoder ökologischen Sicherheit in Studien, deren AutorInnen aus der Entwicklungsoder Umweltschutzbewegung stammen (Busby et al. 2010; Levy et al. 2008; WBGU 2007), unterstreicht die Prädisposition einiger Akteure, bestimmte Frames zu verwenden. Trombetta (2008) verweist in diesem Zusammenhang darauf, dass spezielle Verständnisse von Sicherheit artikuliert werden, um die Argumentation für die Zielgruppe zugänglicher zu gestalten, was sich exemplarisch am Framing von Klimawandel als Gefahr für die nationale Sicherheit der USA zeigt, die vergleichbar sei mit einem Raketenangriff während des Kalten Krieges (CNA 2007: 10).

\subsection{Erfolgsbedingungen}

Die Frage, wer einen spezifischen Frame warum artikuliert, ist eng verbunden mit der Analyse der Erfolgsbedingungen von Versicherheitlichung, da unter anderem die Stellung des versicherheitlichenden Akteurs ausschlaggebend für den Erfolg einer bestimmten Darstellung und ihre Akzeptanz durch das Publikum sein kann. Darüber hinaus wurde die Verwendung einer spezifischen diskursiven Darstellung einer Sicherheitsbedrohung als möglicher Grund für den Erfolg von Versuchen der Versicherheitlichung diskutiert (Guzzini 2011:331). Wir verstehen dabei Erfolg als 
Policy-Relevanz (vgl. Vuori 2008), wobei die Akzeptanz einer diskutierten Maßnahme genügt und diese nicht notwendigerweise bereits umgesetzt sein muss.

Die Forschung zu Framing und Versicherheitlichung identifiziert ähnliche Bedingungen für den Erfolg solcher Prozesse (Snow/Benford 2000; Buzan et al. 1998: 32; Balzacq 2005; Stritzel 2007), die weiteren Aufschluss bezüglich der Frage geben, wie und warum bestimmte Lesarten des Klimawandel-Konflikt-Nexus konkrete Maßnahmen und Programme beeinflussen. Bezug nehmend auf Roxanne L. Dotys (1998) Arbeit zu Migration, stellte McDonald (2008) die Fragen, wie bestimmte Artikulationen von Sicherheit Dominanz erlangten, durch welche Prozesse bestimmte Akteure gestärkt wurden, Sicherheit zu artikulieren und inwieweit bestimmte Lesarten von Sicherheit aufgegriffen beziehungsweise marginalisiert wurden. Anhand der Versicherheitlichung des Klimawandels lässt sich dieser von der Kopenhagener Schule, die den Schwerpunkt der Analyse auf die Folgen statt auf die Prozesse von Versicherheitlichung legt (Wilkinson 2007), nur unzureichend diskutierte Aspekt nachzeichnen.

Drei Bereiche sind in diesem Zusammenhang zentral. Erstens besteht eine größere Erfolgswahrscheinlichkeit, wenn der Sprechakt selbst mit der Grammatik von Sicherheit übereinstimmt (Balzacq 2005; Stritzel 2007). Eine der am häufigsten zitierten Studien zu den Sicherheitsimplikationen des Klimawandels für die nationale Sicherheit der USA diskutiert (fast) ausschließlich die Folgen des Klimawandels für zwischenstaatliche Konflikte und Kriege (Schwartz/Randall 2003). Interessanterweise hat dieser konsistent im Rahmen des Umweltkonflikt-Frames argumentierende Versicherheitlichungsversuch des Klimawandels tatsächlich insofern Politikrelevanz erlangt, als dass daraus resultierende Politikempfehlungen beispielsweise Eingang in den Quadrennial Defense Review Report 2010 des US Department of Defense gefunden haben (Hartmann 2013). Zahlreiche Studien gehen demgegenüber davon aus, dass der fortschreitende Klimawandel vor allem die menschliche Sicherheit von besonders verletzlichen Bevölkerungsgruppen in von den Folgen globaler Erwärmung am schwersten betroffenen Weltregionen gefährdet, ${ }^{6}$ was sich mit dem zunehmenden Fokus auf Anpassungsmaßnahmen deckt (Oels/Carvalho 2012). Dies bedeutet nicht, dass Umweltsicherheitsargumente nicht etwa auch neben Umweltkonfliktargumenten auftauchen können; sie werden letzteren dann allerdings zumeist untergeordnet. Die Sicherheit Einzelner dient in solchen Fällen als Argument für den Ausbau nationaler Sicherheitsvorkehrungen. Die von uns unter-

6 Siehe hierzu exemplarisch die Studie des Wissenschaftlichen Beirats der Bundesregierung Globale Umweltveränderungen (WBGU 2007: 2), in der beispielsweise die Auswirkungen schwindender Süßwasserreserven auf die menschliche Sicherheit und eben nicht auf zwischen- oder innerstaatliche Kriege diskutiert wird. 
suchten Dokumente legen daher nahe, dass Versicherheitlichungsversuche erfolgreicher sind, wenn sie an einem bestimmten Frame festhalten, da dies eine konsistentere Argumentation ermöglicht.

Zweitens ist ein Versuch der Versicherheitlichung erfolgreicher, wenn er existierende Metadiskurse und Weltbilder sowie die Erfahrungen und Ansichten der Zuhörerschaft aufgreift (Balzacq 2005: 171; Léonard/Kaunert 2011). In diesem Kontext wird auch die Rolle von geschichtlichen Narrativen, Kultur und Identität betont (McDonald 2008). Viele der von uns betrachteten Dokumente verwenden das in den USA extrem anschlussfähige Symbol nationaler Sicherheit (Entman 1993: 53-54): So werden beispielsweise sowohl das Überschwappen klimainduzierte Kriege auf die USA (CNA 2007: 1) als auch der unsichere Zugang zu natürlichen Ressourcen aufgrund zunehmender extremer Wetterlagen (Schwartz/Randall 2003: 14) explizit als Gefahr für die nationale Sicherheit dargestellt. Dementsprechend zeigt sich, dass der Grad der Überschneidung zwischen dem jeweiligen Framing und der allgemein vorherrschenden Weltsicht die Chance für eine erfolgreiche Versicherheitlichung beeinflusst. Die »Popularität« des UmweltkonfliktFrames lässt sich demzufolge nicht zuletzt darauf zurückführen, dass diese Lesart des Umwelt-Konflikt-Nexus westliche Verteidigungsinteressen aufgreift und vor dem Hintergrund einer zunehmenden Verschränkung von Sicherheits- und Entwicklungspolitik die Blaupause für eine auch argumentative Verknüpfung beider Bereiche liefert, die beispielsweile in der AFRICOM-Mission (United States Africa Command) ihre Ausgestaltung erfährt (Hartmann 2013: 97; Bettini 2013).

Drittens ist die Position des versicherheitlichenden Akteurs entscheidend. Wie oben diskutiert, geht die Kopenhagener Schule davon aus, dass im stark institutionalisierten Bereich der Sicherheit die politische Elite privilegiert ist, Sicherheitsbedrohungen zu artikulieren (Buzan et al. 1998). Im Gegensatz dazu unterscheidet Thierry Balzacq (2005: 179) zwischen der formalen Position eines versicherheitlichenden Akteurs einerseits und seinem Ansehen als kenntnisreich und vertrauenswürdig andererseits. Darauf aufbauend unterstreicht unsere Analyse, dass der Erfolg eines Versuchs der Versicherheitlichung vom spezifischen Profil eines versicherheitlichenden Akteurs innerhalb einer Gesellschaft abhängt, sowohl hinsichtlich der Organisation, in deren Namen er spricht, als auch hinsichtlich seines eigenen Status. So zeigt sich durch eine Betrachtung der in den Dokumenten verfolgten Argumentationslinien einerseits und ihren AutorInnen sowie der Akzeptanz der Frames andererseits, dass die »diskursive Koalition« (Hajer 1995: 47) ehemaliger Militärs einen nicht unwesentlichen Anteil daran hatte, in den USA den Frame Umweltkonflikt zu verankern (siehe dazu auch Richert 2011). 
Wenig überraschend ist demzufolge, dass die diskursive Verknüpfung von globaler Erwärmung und gewaltsamen Konflikten, die von einflussreichen, dem USVerteidigungsministerium nahestehenden Think Tanks vertreten wird, bei einer bestimmten Zuhörerschaft eine größere Akzeptanz erlangt haben als die von kleinen, kapitalismuskritischen NGOs vertretenen Argumentationen im Rahmen des Frames der ökologischen Sicherheit. Andererseits wurde in Bolivien, wo 2010 die erste Weltkonferenz der Völker über den Klimawandel und die Rechte der »Mutter Erde « stattfand, entsprechend der Sichtweise, dass der Klimawandel die »Mutter Erde« als solche gefährdet, ein Umweltschutzgesetz verabschiedet, das die »Mutter Erde« als eigenständiges Rechtssubjekt anerkennt (Schilling-Vacaflor/Barrera 2011). Dies unterstreicht die Notwendigkeit, unterschiedliche Zuhörerschaften in die Analyse einzubeziehen.

Eine vierte Bedingung kann, der Diskussion von Versicherheitlichung durch die Pariser Schule (Bigo 2000; 2002) folgend, hinzugefügt werden. Dementsprechend ist der Erfolg einer Versicherheitlichung beeinflusst von der zu erwartenden Realisierbarkeit, was 1.) die technologischen Möglichkeiten, 2.) die Umsetzung in administrative Praktiken und 3.) die erwarteten Kosten anbelangt. Während die Kopenhagener Schule solche Faktoren vernachlässigt, indem sie den Ausnahmecharakter der gegen die Sicherheitsbedrohung ergriffenen Maßnahmen betont, vertreten wir die Auffassung, dass auch solche technokratischen Praktiken außergewöhnliche Maßnahmen darstellen können. Nichtsdestotrotz stützen die Dokumente die Annahme, dass Versicherheitlichungsversuche eher Erfolg haben, wenn die Kosten dafür relativ niedrig sind und Maßnahmen vorgeschlagen werden, die ohne Weiteres verfügbar sind, um die diagnostizierte Sicherheitsbedrohung zu bekämpfen. Dementsprechend diskutieren beispielsweise nur zwei Dokumente Geoengineering, und zwar vor allem im Zusammenhang mit zu hohen Kosten und technologischen Risiken (Campbell et al. 2007: 18; etwas positiver Schwartz/Randall 2003: $3,22)$.

Es ist zwar richtig, dass eine erfolgreiche Versicherheitlichung im Sinne der Kopenhagener Schule zu außergewöhnlichen Maßnahmen - »koste es, was es wolle« - führen sollte. Zu bedenken gilt es aber, dass selbst während des Kalten Krieges diesen Maßnahmen budgetäre und technologische Grenzen gesetzt waren. Methmann und Rothe (2012) verweisen darauf, dass im Bereich des Klimawandels bisher eher technokratische und als »realistisch « charakterisierte Problembearbeitungen dominieren, die jedoch - anders als von den Autoren postuliert - nicht nur der Ausfluss apokalyptischer Problemdiagnosen sind. In den betrachteten Dokumenten werden Forderungen nach einer eher inkrementellen und bruchstückhaften Veränderung der Governance-Architektur vor allem laut, um besser auf die Implikationen 
des Klimawandels für die menschliche Sicherheit reagieren zu können (exemplarisch hierfür WBGU 2007).

\section{Der Mehrwert eines Framing-Ansatzes}

Wir haben in diesem Artikel gezeigt, dass es verschiedene Arten der Versicherheitlichung des Klimawandels gibt, die Inhalte dieser verschiedenen Frames dargelegt und die politischen Konsequenzen aufgezeigt, die diese hervorrufen. Um die Defizite der Kopenhagener Schule der Versicherheitlichung bei der Analyse des Klimawandel-Sicherheits-Nexus zu beheben, haben wir vorgeschlagen, Versicherheitlichungen als Framing-Prozesse zu begreifen. Dies erlaubte es uns, drei verschiedene Frames zu identifizieren, in deren Rahmen der Klimawandel versicherheitlicht wird und die jeweils verschiedene normative Probleme zur Folge haben. Eine nuancierte Betrachtung der verschiedenen Frames problematisiert darüber hinaus die Vorstellung, die Sicherheitsimplikationen des Klimawandels bestünden (ausschließlich) in einem erhöhten Konflikt- und (Bürger-)Kriegsrisiko.

Vier zentrale Erkenntnisse ergeben sich aus dieser Analyse. Eine kritische Lektüre einflussreicher Berichte bestätigt einen Zusammenhang zwischen der diagnostischen Dimension, d. h. der (kausalen) Zuschreibung von Problemen und Referenzobjekten auf der einen Seite und der prognostischen Dimension, also den vorgeschlagenen politischen Maßnahmen auf der anderen Seite. So spielte eine militärische Anpassung in Studien, die den Klimawandel als Auslöser oder Multiplikator bewaffneter Konflikte diskutieren (Schwartz/Randall 2003; CNA 2007; Campbell et al. 2007), eine zentrale Rolle.

Zweitens sind Versuche, den Klimawandel zu versicherheitlichen, von FrameTransformationen und Frame-Auseinandersetzungen geprägt. Während einige AutorInnen eine graduelle Verschiebung von einer Betonung der Gefahr für die menschliche Sicherheit hin zu einem Fokus auf inner- und zwischenstaatliche Klimakonflikte nicht bestätigt sehen (Detraz/Betsill 2008), konstatieren andere eine solche Entwicklung, vor allem im Zusammenhang mit Versuchen, den Klimawandel auf eine für US-Außen- und SicherheitsexpertInnen relevante Weise zu thematisieren (Mayer 2012). Insbesondere hinsichtlich der Frage, auf welche Weise die fortschreitende globale Erwärmung Sicherheitsimplikationen mit sich bringt, herrscht Uneinigkeit, wobei die AutorInnen einiger Studien einen - aus ihrer Sicht - verengten Fokus auf Klimakonflikte als diagnostisch falsch ablehnen (WBGU 2007).

Drittens ermöglicht ein Framing-Ansatz, die von der Kopenhagener Schule entwickelte und als konzeptionell problematisch betrachtete sowie für die empirische 
Analyse unzureichende Dichotomie zwischen »normaler « Politik und auf erfolgreicher Versicherheitlichung basierender Notfallmaßnahmen auszudifferenzieren. Abhängig von der jeweiligen Problemdiagnose kann auch eine darauf reagierende drastische Umgestaltung der weltweiten Umwelt-Governance oder eine anderenfalls nicht in Betracht gezogene Begrenzung der Treibhausgasemissionen eine außergewöhnliche Maßnahme darstellen, die auf bestimmte Sicherheitsimplikationen des Klimawandels reagiert.

Schließlich hat die Abkehr von der Politik/Sicherheit-Dichotomie auch Auswirkungen auf die Debatte über die normativen Konsequenzen von Versicherheitlichung. Eine Konzeptualisierung von Versicherheitlichung als Framing-Prozess kann dem »normative dilemma of speaking and writing security« (Huysmans 1999) nicht entgegenwirken. Nichtsdestotrotz können die verschiedenen Frames der Versicherheitlichung des Klimawandels ein differenzierteres Bild der normativen Implikationen des Klimawandels zeichnen, dessen problematische Folgen in bestimmten diskursiven Darstellungen prononcierter sind als in anderen. Dies bedeutet, dass Versuche der Versicherheitlichung, die große Übereinstimmung mit der von Wæver (1995) definierten linguistischen Struktur aufweisen - sofern sie erfolgreich sind eher zur Versicherheitlichung als zur Politisierung führen. Im konkreten Fall münden die Frames Umweltsicherheit und ökologische Sicherheit aber eher in eine Politisierung der Debatte, verstanden als eine Zunahme argumentativer Positionen, während das Frame Umweltkonflikte eher zu einer normativ problematischen Versicherheitlichung im Sinne einer Militarisierung oder Positionsverengung führt.

Ebenso zeigt Floyd (2008; 2010), dass einige Ansätze der Versicherheitlichung von Umweltproblemen zu einer schnellen und effektiven Lösung im Rahmen eines politischen Prozesses führten, statt eine Logik der Konfrontation zu provozieren. Sie argumentiert folglich, dass Versicherheitlichungen a priori weder positiv noch negativ sind, sondern vielmehr entsprechend ihrer politischen Ergebnisse beurteilt werden müssen. Während ihre Arbeit erste Eindrücke bietet, kann nur eine systematische Studie von Versuchen der Versicherheitlichung, die unterschiedliche diskursive Frames in einem bestimmten Sektor anwenden, zeigen, ob und wie das exakte Framing die Auswirkungen bezüglich Versicherheitlichung und Politisierung beeinflusst. Dies verweist auf die grundlegende Frage, welche Rolle Versicherheitlichung bei der Politisierung von Themen spielt. Trombetta (2011: 145) argumentiert hierzu bezogen auf das Ozonregime, dass die Politisierung des Sachverhalts durch seine Versicherheitlichung erfolgte. Eine systematische Analyse der Möglichkeit eines solchen Prozesses bei der Versicherheitlichung des Klimawandels steht noch aus. Es erscheint daher lohnenswert, in künftigen Studien genauer 
zu untersuchen, welche Rolle unterschiedliche Framings von Klimawandel als Sicherheitsbedrohung spielen.

\section{Literatur}

Adamides, Constaninos 2012: Institutionalized, Horizontal and Bottom-Up Securitization in Ethnic Conflict Environments. The Case of Cyprus (University of Birmingham, $\mathrm{PhD}$ Thesis), in: http://etheses.bham.ac.uk/3791/1/ Adamides_PhD_12.pdf; 5.3.2013.

Adger, Neil $W$. 2006: Vulnerability, in: Global Environmental Change 16: 3, 268-281.

Balzacq, Thierry 2005: The Three Faces of Securitization. Political Agency, Audience and Context, in: European Journal of International Relations 11:2, 171-201.

Balzacq, Thierry 2011: A Theory of Securitization. Origins, Core Assumptions, and Variations, in: Balzacq, Thierry (Hrsg.): Securitization Theory. How Security Problems Emerge and Dissolve, New York, NY, 1-30.

Barnett, Jon 2001: The Meaning of Environmental Security, London.

Benford, Robert D. 1993: Frame Disputes within the Nuclear Disarmament Movement, in: Social Forces 71: 3, 677-701.

Benford, Robert D./Snow, David A. 2000: Framing Processes and Social Movements. An Overview and Assessment, in: Annual Review of Sociology Vol. 26, 611-639.

Bertell, Rosalie 2001: Planet Earth. The Latest Weapon of War, Montreal.

Bettini, Giovanni 2013: (In)convenient Convergence: ১Climate Refugees`, Apocalyptic Discourses and Depoliticisation of Climate-induced Migration, in: Methmann, Chris/Rothe, Delf/Stephan, Benjamin (Hrsg.): Interpretive Approaches to Global Climate Governance. (De)constructing the Greenhouse, Abingdon, 122-136.

Biermann, Frank/Boas, Ingrid 2008: Protecting Climate Refugees. The Case for a Global Protocol, in: Environment 50: 6, 8-16.

Bigo, Didier 2000: When Two Become One. Internal and External Securitisations in Europe, in: Kelstrup, Morten/Williams, Michael C. (Hrsg.): International Relations Theory and the Politics of European Integration, London, 171-204.

Bigo, Didier 2002: Security and Immigration. Towards a Critique of the Governmentality of Unease, in: Alternatives: Global, Local, Political 27: 1, 63-92.

Brzoska, Michael 2009: The Securitization of Climate Change and the Power of Conceptions of Security, in: Security and Peace 27: 3, 137-145. 
Buck, Holly J. 2013: Climate Engineering. Spectacle, Tragedy or Solution? A Content Analysis of News Media Framing, in: Methmann, Chris/Rothe, Delf/ Stephan, Benjamin (Hrsg.): Interpretive Approaches to Global Climate Governance. (De)constructing the Greenhouse, Abingdon, 166-180.

Burke, M. B./Miguel, E./Satyanath, S./Dykema, J. A./Lobell, D. B. 2009: Warming Increases the Risk of Civil War in Africa. Proceedings of the National Academy of Sciences 106: 49, 20670-20674.

Busby, Joshua W. 2007: Climate Change and National Security. An Agenda for Action (Council Special Report (CSR) No. 32), in: http://i.cfr.org/content/publications/attachments/ClimateChange_CSR32.pdf; 13.10.2011.

Busby, Joshua W./Gulledge, Jay/Smith, Todd/White, Kaiba 2010: Of Climate Change and Crystal Balls. The Future Consequences of Climate Change in Africa, in: http://ccaps.strausscenter.org/system/research_items/pdfs/30/original.pdf?1283893878; 16.10.2011.

Busse, Dietrich/Teubert, Wolfgang 1994: Ist Diskurs ein sprachwissenschaftliches Objekt? Zur Methodenfrage der Historischen Semantik, in: Busse, Dietrich/ Hermanns, Fritz/Teubert, Wolfgang (Hrsg.): Begriffsgeschichte und Diskursgeschichte. Methodenfrage und Forschungsergebnisse der Historischen Semantik, Opladen, 10-28.

Buzan, Barry/Waver, Ole/De Wilde, Jaap 1998: Security. A New Framework for Analysis, Boulder, CO.

Buzan, Barry/Woever, Ole 2009: Macro-securitisations and Security Constellations. Reconsidering Scale in Securitisation Theory, in: Review of International Studies 35: 2, 252-276.

Campbell, Kurt M./Gulledge, Jay/McNeill, J.R/Podesta, John/Ogden, Peter/Fueth, Leon/Woolsey, R. James/Lennon, Alexander T.J./Smith, Julianne/Weitz, Richard/Mix, Derek 2007: The Age of Consequences. Foreign Policy and National Security Implications of Global Climate Change, in: http://csis.org/files/media/ csis/pubs/071105_ageofconsequences.pdf; 13.10.2011.

Carragee, Kevin M./Roefs, Wim 2004: The Neglect of Power in Recent Framing Research, in: Journal of Communication 54: 2, 214-233.

Center for Naval Analyses - CNA 2007: National Security and the Threat of Climate Change, in: http://www.cna.org/sites/default/files/National\%20Security \%20and\%20the\%20Threat\%20of\%20Climate\%20Change.pdf; 30.3.2011.

Corry, Olaf 2012: Securitisation and »Riskification«: Second-Order Security and the Politics of Climate Change, in: Millennium: Journal of International Studies 40: 2, 235-58. 
Cudworth, Erika/Hobden, Stephen 2010: Securing What for Whom? Multiple Complex Inequalities and the Politics of Environmental Security in Europe (paper presented to the ECPR $5^{\text {th }}$ Pan European Conference on the EU, Porto, Portugal, 24.-26.6.2010), in: http://www.jhubc.it/ecpr-porto/virtualpaperroom/021.pdf, 25.9.2014.

Daase, Christopher 2009: Der erweiterte Sicherheitsbegriff, in: Ferdowsi, Mir A. (Hrsg.): Internationale Politik als Überlebensstrategie, München, 137-153.

Dalby, Simon 1992: Ecopolitical Discourse. Environmental Security and Political Geography, in: Progress in Human Geography 16: 4, 503-522.

Dalby, Simon 2000: Geopolitical Change and Contemporary Security Studies. Contextualizing the Human Security Agenda (Institute of International Relations. The University of British Columbia Working Paper No. 30), in: http://se2.isn.ch/ serviceengine/Files/RESSpecNet/46517/ipublicationdocument_singledocument/ 9B234690-56E8-4DF4-87ED-B614DD6C2A4B/en/WP30.pdf; 1.3.2011.

Detraz, Nicole/Betsill, Michele M. 2009: Climate Change and Environmental Security. For Whom the Discourse Shifts, in: International Studies Perspectives 10: 3, 303-320.

Doty, Roxanne L. 1998: Immigration and the Politics of Security, in: Security Studies 8: 2-3, 71-93.

Eakin, Hallie/Luers, Amy L. 2006: Assessing the Vulnerability of Social-Environmental Systems, in: Annual Review of Environment and Resources 31, 365-394. Elbe, Stefan 2005: AIDS, Security and Biopolitics, in: International Relations 19: 4, 403-419.

Entman, Robert M. 1993: Framing. Toward Clarification of a Fractured Paradigm, in: Journal of Communication 43: 4, 51-58.

Eriksson, Johan (Hrsg.) 2001: Threat Politics. New Perspectives on Security, Risk, and Crisis Management, Aldershot.

Floyd, Rita 2008: The Environmental Security Debate and Its Significance for Climate Change, in: The International Spectator 43: 3, 51-65.

Floyd, Rita 2010: Security and the Environment. Securitization Theory and US Environmental Security Policy, Cambridge.

Flyvbjerg, Bent 2006: Five Misunderstandings About Case-Study Research, in: Qualitative Inquiry 12: 2, 219-245.

Gerhards, Jürgen/Rucht, Dieter 1992: Mesomobilization. Organization and Framing in Two Protest Campaigns in West Germany, in: American Journal of Sociology 98: 3, 555-595.

Glynos, Jason/Howarth, David 2007: Logics of Critical Explanation in Social and Political Theory, Abington. 
Goffman, Erving 1974: Frame Analysis. An Essay on the Organization of Experience, Cambridge, MA.

Guzzini, Stefano 2011: Securitization as a Causal Mechanism, in: Security Dialogue 42: 4-5, 329-341.

Hajer, Maarten A. 1995: The Politics of Environmental Discourse. Ecological Modernization and the Policy Process, Oxford.

Hartmann, Betsy 2009: From Climate Refugees to Climate Conflict. Who Is Taking the Heat for Global Warming?, in: Salih, Mohamed (Hrsg.): Climate Change and Sustainable Development. New Challenges for Poverty Reduction, Cheltenham, 141-155.

Hartmann, Betsy 2013: Climate Chains. Neo-Malthusianism, Militarism and Migration, in: Methmann, Chris/Rothe, Delf/Stephan, Benjamin (Hrsg.): Interpretive Approaches to Global Climate Governance. (De)constructing the Greenhouse, Abingdon, 91-104.

Holling, Crawford S. 1973: Resilience and Stability of Ecological Systems, in: Annual Review of Ecology and Systematics Vol. 4, 1-23.

Homer-Dixon, Thomas 1994: Environmental Scarcity and Violent Conflicts. Evidence from Cases, in: International Security 19: 1, 5-40.

Homer-Dixon, Thomas 1999: Environment, Scarcity and Conflict, Princeton, NJ.

Hulme, Mike 2010: Problems with Making and Governing Global Kinds of Knowledge, in: Global Environmental Change 20: 4, 558-564.

Huysmans, Jef 1998: Revisiting Copenhagen: Or, on the Creative Development of a Security Studies Agenda in Europe, in: European Journal of International Relations 4: 4, 479-505.

Huysmans, Jef 1999: Language and the Mobilization of Security Expectations. The Normative Dilemma of Speaking and Writing Security (Konferenzpapier vorgestellt bei den ECPR Joint Sessions 26.-31.5.1998), Mannheim.

Huysmans, Jef 2006: The Politics of Insecurity. Fear, Migration and Asylum in the EU, London.

Jäger, Siegfried 2006: Diskurs und Wissen. Theoretische und Methodische Aspekte einer Kritischen Diskurs- und Dispositivanalyse, in: Keller, Reiner/Hirseland, Andreas/Schneider, Werner/Viehöver, Willy (Hrsg.): Handbuch Sozialwissenschaftliche Diskursanalyse. Band 2: Forschungspraxis, Wiesbaden, 83-114.

Kaplan, Robert 1994: The Coming Anarchy, in: The Atlantic Monthly 273:2, 44-76. Léonard, Sarah/Kaunert, Christian 2011: Reconceptualizing the Audience in Securitization Theory, in: Balzacq, Thierry (Hrsg.): Securitization Theory. How Security Problems Emerge and Dissolve, Abingdon, 57-76. 
Levy, Marc A./Anderson, Bridget/Brickman, Melanie/Cromer, Chris/Falk, Brian/ Fekete, Balazs/Green, Pamela/Jaiteh, Malanding/Lammers, Richard/Mara, Valentina/MacManus, Kytt/Metzler, Steve/Muñiz, Maria/Parris, Thomas/Pullen, Randy/Thorkelson, Catherine/Vorosmarty, Charles/Wollheim, Wil/Xing, Xiaoshi/Yetman, Greg 2008: Assessment of Select Climate Change Impacts on U.S. National Security (Center for International Earth Science Information Network Working Paper), in: http://www.ciesin.columbia.edu/documents/Climate_ Security_CIESIN_July_2008_v 1_0.ed.pdf; 21.10.2014.

Litfin, Karen T. 1994: Ozone Discourses. Science and Politics in Global Environmental Cooperation, New York, NY.

MacKinnon, Danny/Derickson, Kate 2013: From Resilience to Resourcefulness: A Critique of Resilience Policy and Activism, in: Progress in Human Geography 37, 251-268.

Malone, Linda A. 1996: Green Helmets. A Conceptual Framework for Security Council Authority in Environmental Emergencies (Faculty Publications Paper 599), in: http://scholarship.law.wm.edu/facpubs/599; 25.11.2013.

Mayer, Maximilian 2012: Chaotic Climate Change and Security, in: International Political Sociology 6: 2, 165-185.

McDonald, Matt 2008: Securitization and the Construction of Security, in: European Journal of International Relations 14: 4, 563-587.

McDonald, Matt 2013: Discourses of Climate Security, in: Political Geography Vol. 33, 42-51.

Methmann, Chris/Rothe, Delf 2012: Politics for the Day after Tomorrow. The Logic of Apocalypse in Global Climate Politics, in: Security Dialogue 44: 5-6, 323-344.

Methmann, Chris/Rothe, Delf 2013: Apocalypse Now, in: Methmann, Chris/Rothe, Delf/Stephan, Benjamin (Hrsg.): Interpretive Approaches to Global Climate Governance. (De)constructing the Greenhouse, Abingdon, 105-121.

Methmann, Chris/Rothe, Delf/Stephan, Benjamin 2013: Introduction. How and Why we Construct the Greenhouse, in: dies. (Hrsg.): Interpretive Approaches to Global Climate Governance: (De)constructing the Greenhouse, Abingdon, 1-22.

Neal, Andrew W. 2009: Securitization and Risk at the EU Border: The Origins of FRONTEX, in: Journal of Common Market Studies 47: 2, 333-356.

Neverla, Irene/Schäfer, Mike S. 2010: Das Medienklima. Relevanz und Spezifika der Medienberichterstattung über den anthropogenen Klimawandel, in: Mitteilungen der Deutschen Meteorologischen Gesellschaft 3/2010, 9-12. 
Neverla, Irene/Schäfer, Mike S. 2012: Einleitung. Der Klimawandel und das Medienklima, in: dies. (Hrsg.): Das Medien-Klima. Fragen und Befunde der kommunikationswissenschaftlichen Klimaforschung, Wiesbaden, 9-25.

Nisbet, Matthew C. 2009: Communicating Climate Change: Why Frames Matter for Public Engagement, in: Environment: Science and Policy for Sustainable Development 51: 2, 12-23.

Nordås, Ragnhild/Gleditsch, Nils Petter 2007: Climate Change and Conflict, in: Political Geography 26: 6, 627-638.

Oels, Angela/Carvalho, Anabela 2012: Wer hat Angst vor »Klimaflüchtlingen«? Wie die mediale und politische Konstruktion des Klimawandels den politischen Handlungsraum strukturiert, in: Neverla, Irene/Schäfer, Mike S. (Hrsg.): Das Medien-Klima. Fragen und Befunde der kommunikationswissenschaftlichen Klimaforschung, Wiesbaden, 253-276.

Page, Edward 2010: What's the Point of Environmental Security? (Papier vorgestellt auf der SGIR $7^{\text {th }}$ Pan-European International Relations Conference, 11.9.2010), Stockholm.

Pram Gad, Ulrik/Petersen, Karen L. 2011: Concepts of Politics in Securitization Studies, Security Dialogue 42: 4-5, 315-328.

Reese, Stephen D. 2007: The Framing Project. A Bridging Model for Media Research Revisited, in: Journal of Communication 57: 1, 148-154.

Rein, Martin/Schön, Donald 1991: Frame Reflective Policy Discourses, in: Wagner, Peter/Weiss, Carol Hirschon/Wittrock, Björn/Wollmann, Hellmut (Hrsg.): Social Science and Modern States. National Experiences and Theoretical Crossroads, Cambridge, 262-289.

Richert, Jörn 2011: Klimawandel, Bedrohungsdiskurs und Sicherheitspolitik in den USA, in: Angenendt, Steffen/Dröge, Susanne/Richert, Jörn (Hrsg.): Klimawandel und Sicherheit. Herausforderungen, Reaktionen und Handlungsmöglichkeiten, Baden-Baden, 222-237.

Ross, Michael L. 2003: The Natural Resource Curse. How Wealth Can Make You Poor, in: Bannon, Ian/Collier, Paul (Hrsg.): Natural Resources and Violent Conflict. Options and Actions, Washington, D.C., 17-42.

Ross, Michael L. 2004: What Do We Know about Natural Resources and Civil War?, in: Journal of Peace Research 41: 3, 337-356.

Salter, Mark B. 2008: Securitization and Desecuritization. A Dramaturgical Analysis of the Canadian Air Transport Security Authority, in: Journal of International Relations and Development 11: 4, 321-349. 
Salter, Mark B. 2011: When Securitization Fails. The Hard Case of Counter-Terrorism Programs, in: Balzacq, Thierry (Hrsg.): Securitization Theory. How Security Problems Emerge and Dissolve, Abingdon, 116-132.

Scheufele, Dietram A. 2000: Agenda Setting, Priming and Framing Revisited. Another Look at Cognitive Effects of Political Communication, in: Mass Communication and Society 3: 2-3, 297-316.

Schilling-Vacaflor, Almut/Barrera, Anna 2011: Lateinamerikas neue Verfassungen. Triebfedern für direkte Demokratie und soziale Rechte? (GIGA Focus 2/2011), in: http://www.giga-hamburg.de/de/system/files/publications/gf_lateinamerika 1102.pdf; 25.11.2013.

Schmidt, Andreas 2012: Bewegungen, Gegenbewegungen, NGOs. Klimakommunikation zivilgesellschaftlicher Akteure, in: Neverla, Irene/Schäfer, Mike S. (Hrsg.): Das Medien-Klima. Fragen und Befunde der kommunikationswissenschaftlichen Klimaforschung, Wiesbaden, 69-94.

Schwartz, Peter/Randall, Doug 2003: An Abrupt Climate Change Scenario and Its Implications for United States National Security, in: http://oco.jpl.nasa.gov/pu bs/Abrupt_Climate_Change_Scenario.pdf; 1.3.2011.

Sjostedt, Roxanna 2011: Health Issues and Securitization. The Construction of HIV/ AIDS as a US National Security Threat, in: Balzacq, Thierry (Hrsg.): Securitization Theory. How Security Problems Emerge and Dissolve, London, 150-169. Smith, Michael E. 2010: International Security. Politics, Policy, Prospects, Houndmills.

Smith, Dan/Vivekananda, Janani 2007: A Climate of Conflict: The Links between Climate Change, Peace and War, in: http://www.international-alert.org/sites/default/files/publications/A_climate_of_conflict.pdf; 11.7.2011.

Stritzel, Holger 2007: Towards a Theory of Securitization: Copenhagen and Beyond, in: European Journal of International Relations 13: 3, 357-383.

Stritzel, Holger 2011: Security, the Translation, in: Security Dialogue 42: 4-5, 343-355.

Tarrow, Sidney 1982: National Politics and Collective Action. Recent Theory and Research in Western Europe and the United States, in: Annual Review of Sociology Vol. 14, 421-440.

Trombetta, Maria J. 2008: Environmental Security and Climate Change. Analysing the Discourse, in: Cambridge Review of International Affairs 21: 4, 585-602.

Trombetta, Maria J. 2011: Rethinking the Securitization of the Environment: Old Beliefs, New Insights, in: Balzacq, Thierry (Hrsg.): Securitization Theory. How Security Problems Emerge and Dissolve, London, 135-149. 
Ullmann, Richard 1983: Redefining Security, in: International Security 8: 1, 129-153.

Verhoeven, Harry 2011: Climate Change, Conflict and Development in Sudan. Global Neo-Malthusian Narratives and Local Power Struggles, in: Development and Change 42: 3, 679-707.

Vogel, Coleen/O'Brien, Karen 2004: Vulnerability and Global Environmental Change. Rhetoric and Reality, in: AVISO 13, 1-8.

Vultee, Fred 2010: Securitization as a Media Frame, in: Balzacq, Thierry (Hrsg.): Securitization Theory. How Security Problems Emerge and Dissolve, London, 77-93.

Vuori, Juha A. 2008: Illocutionary Logic and Strands of Securitization. Applying the Theory of Securitization to the Study of Non-Democratic Political Orders, in: European Journal of International Relations 14: 1, 65-99.

Wever, Ole 1995: Securitization and Desecuritization, in: Lipschutz, Ronnie D. (Hrsg.): On Security, New York, NY, 46-86.

Walker, Jeremy/Cooper, Melinda 2011: Genealogies of Resilience. From Systems Ecology to the Political Economy of Crisis Adaptation, in: Security Dialogue 42: 2, 143-160.

Wissenschaftlicher Beirat der Bundesregierung Globale Umweltveränderungen WBGU 2007: Welt im Wandel. Sicherheitsrisiko Klimawandel, in: http://www. wbgu.de/fileadmin/templates/dateien/veroeffentlichungen/hauptgutachten/jg20 07/wbgu_jg2007.pdf; 1.3.2011.

Wilkinson, Claire 2007: The Copenhagen School on Tour in Kyrgyzstan. Is Securitization Theory Useable Outside Europe?, in: Security Dialogue 38: 5, 5-25.

Williams, Michael C. 2003: Words, Images, Enemies. Securitization and International Politics, in: International Studies Quarterly 47: 4, 511-531.

Williams, Michael C. 2011: The Continuing Evolution of Securitization Theory, in: Balzacq, Thierry (Hrsg.): Securitization Theory. How Security Problems Emerge and Dissolve, London, 212-222.

Zhang, David D./Brecke, Peter/Lee, Harry F./He, Yuan-Qing/Zhang, Jane 2007:

Global Climate Change, War, and Population Decline in Recent Human History, in: Proceedings of the National Academy of Sciences of the United States of America 104: 49, 19214-19219. 


\section{Die AutorInnen}

Julia Grauvogel ist Research Fellow am German Institute of Global and Area Studies (GIGA). E-Mail: julia.grauvogel@giga-hamburg.de

Prof. Dr. Thomas Diez ist Professor für Politikwissenschaft und Internationale Beziehungen an der Eberhard-Karls-Universität Tübingen.

E-Mail: thomas.diez@uni-tuebingen.de 